\title{
Michael Lucken, 1945 Hiroshima. Les images sources
}

Paris, Hermann, 2008

\section{Tanguy Wuillème}

\section{(2) OpenEdition}

\section{Journals}

Édition électronique

URL : http://journals.openedition.org/questionsdecommunication/946

DOI : 10.4000/questionsdecommunication.946

ISSN : 2259-8901

\section{Éditeur}

Presses universitaires de Lorraine

\section{Édition imprimée}

Date de publication : 1 juillet 2009

Pagination : 448-451

ISBN : 978-2-86480-989-0

ISSN : $1633-5961$

\section{Référence électronique}

Tanguy Wuillème, " Michael Lucken, 1945 Hiroshima. Les images sources », Questions de communication [En ligne], 15 | 2009, mis en ligne le 17 janvier 2012, consulté le 21 septembre 2020. URL : http:// journals.openedition.org/questionsdecommunication/946 ; DOI : https://doi.org/10.4000/ questionsdecommunication.946

Ce document a été généré automatiquement le 21 septembre 2020

Tous droits réservés 


\section{Michael Lucken, 1945 Hiroshima. Les images sources}

Paris, Hermann, 2008

Tanguy Wuillème

\section{RÉFÉRENCE}

Michael Lucken, 1945 Hiroshima. Les images sources. Paris, Hermann, 2008,199 p.

Dans son roman L'immortalité (trad. du tchèque par Éva Bloch, Paris, Gallimard, 1990), Milan Kundera affirme que "la mémoire ne filme pas, la mémoire photographie " (p. 83). Si l'Histoire épouse le mouvement des êtres et des choses, la mémoire comporte un aspect statique, elle engrange des images avec lesquelles il est difficile de reconstruire le film. Les manuels d'histoire n'ont longtemps montré de l'explosion des bombes atomiques d'Hiroshima et de Nagasaki qu'un champignon grossi dans un ciel dégagé. Le point de vue est celui du bourreau bien assis dans sa chaise, de la distance analytique, de la caricature journalistique. Tout l'intérêt de ce livre est d'évaluer la qualité de l'expérience d'un fait qui a bouleversé l'humanité et qui, bien souvent, n'a pas profité du travail des historiens et des spécialistes de l'image. En comparaison de la solution finale ou des camps de concentration soviétiques, des génocides arménien, balkanique, rwandais... ce qui s'est passé, ce qui a été vécu et vu au mois d'août 1945 n'a pas été analysé, ni compris. Il ne s'agit pas d'opposer les mémoires entre elles, mais de poursuivre l'investigation plus en avant et de voir pourquoi à Hiroshima plus qu'ailleurs, un autre statut a été donné au témoignage et au regard.

2 Comme l'indique Michael Lucken dans son préambule, les rapports à la destruction étaient autrefois inscrits dans un cadre rendant progressive l'appréhension de celle-ci. César ou Napoléon prenaient le temps d'apprécier le déroulé d'une bataille. En 1945, au Nouveau Mexique, lors du premier test nucléaire, les officiers et les scientifiques présents se verront donner l'ordre de détourner le regard. Seuls des outils techniques ultra perfectionnés seront à même de calculer l'événement. Cela indique que 
l'expérience est devenue impossible, qu'elle excède les capacités et catégories humaines de compréhension. Aucun plan spatial et temporel ne pourrait servir d'index, de repères pour l'homme, seuls ses médias permettent une expérience décisive. Si nous ne sommes plus à la hauteur de nos machines, de quoi peut-on encore témoigner ? À Hiroshima, le témoin oculaire a pu ne rien voir ou, comme tout événement, a pu ne voir qu'une parcelle déformée de la réalité. Les images lacunaires ont pu être mises en discours, en films, en poèmes, mais comment retrouver la véritable teneur de ce qui s'est passé? L'hypothèse de l'ouvrage est que la photographie constitue une porte d'entrée non négligeable. Elle retrouve le temps et le recul physique, elle serait plus conforme à nos capacités mémorielles, à nos réactions sensorielles, à nos cadres psychophysiologiques. Les pensées et souvenirs marchent à l'image. Toutefois, celle de la destruction demande une attention autre, plus soutenue et plus lente.

Il faut déplacer ces images des espaces où elles figurent habituellement: les mémoriaux, les livres d'histoire, les actualités, même si tous tentent de perpétuer et de transmettre l'expérience. Le livre offre ici un format rectangulaire où l'on peut apprécier tranquillement les photographies qui ne sont pas empilées, mais entrecoupées par le commentaire (notons cependant que la reliure collée est de faible consistance, ce qui pour un livre de photo augure mal de sa conservation). On voit combien l'événement a été lui-même photographique :un immense flash qui a brûlé les corps et n'a gardé d'eux que l'ombre portée, fixée sur les monuments. La déflagration atomique a comme produit une immense photographie in vivo, sa plaque d'impression fut les objets, le monde environnant, ses sujets furent au fond ce qu'ils étaient dans l'esprit des Américains, des ombres qui fallaient réduire en tant que telles, en poussière objectale.

4 L'armée des États-Unis fit aussi un autre emploi de la photographie : c'est grâce à elle qu'elle pût préparer l'attaque sur le Japon, ne cessant de survoler le territoire pour mieux en repérer les sites et les cibles: "La photographie fut, au même titre que les radars, un instrument indispensable des campagnes américaines de bombardements massifs » (p. 29). Instrument de repérages, de précision, la photographie fut avant tout un appareillage militaire assurant là encore la réussite de l'ère technique. Ce sont ces images que la presse reprend le plus souvent, sans en interroger le point de vue ni la source. Néanmoins, les principales photographies de prises de vue au sol qui suivirent l'événement sont toutes d'origine japonaise. Elles sont le fait de professionnels accrédités, autorisés militairement à se rendre sur les lieux. Que ce soit celles de Matsushige Yoshito ou celles de Yamahata Yosuke, ces images sont issues d'une culture du reportage de guerre et de la propagande et comme telles soumises à l'intériorisation des normes de la censure. On pense aujourd'hui qu'il y aurait eu environ 2000 clichés provenant d'une quinzaine de photographes, on n'en possède plus que 230, les autres ayant disparu ou été détruits (effet de la radioactivité et contrôle des autorités occupantes). Ces clichés sont eux-mêmes des survivants au destin exceptionnel. Trois sujets y sont représentés : des scènes de ruines, la colonne de fumée et seulement une cinquantaine sur les blessés. Très rares sont ceux qui montrent des cadavres, ce qui confirme l'autocensure (évitement des aspects négatifs et de la défaite), mais aussi la difficulté à appréhender la mort à l'œuvre. L'auteur constate que ces photographies de cadavres ne sont jamais mises au cœur du dispositif commémoratif et sont peu reproduites. Les voir est terrifiant: visages hébétés tachetés, corps décharnés où le squelette apparait et surtout ces photographies d'enfants noircis, comme sombrement 
plastifiés, tombés à côté de leurs mères. On n'y voit guère de soldats puisque les civils furent visés.

5 Les images que l'auteur dit ratées nous ont au contraire semblé pertinentes. De l'ensemble des premières images de familles abattues sur les trottoirs, recroquevillées, prostrées, encore vivantes dans une ambiance noire et sale, on perçoit l'humanité qui se relève dans la poussière, qui reprend vie dans le chaos. Le visage n'y est pas présent parce que sa couleur se confond avec le reste, parce qu'il est lui-même devenu corps. Mais ces photographies apportent la contradiction de villes devenues des déserts uniformes: il reste de l'humain, même si c'est sous forme de déchets. Il ne s'agit aucunement d'une table rase.

Ces visages, on ne les retrouvera jamais dans les photographies d'après-guerre. Ce qui sera reconstruit consistera uniquement en la surface de la peau, le cuir chevelu, les membres défaits. La greffe ou la prothèse seront les signes et la reconstruction même du pays. Là encore, il était impossible de regarder la réalité en face. On sait que le Japon entretient toujours une forme d'interdit sur la représentation de la mort atomique (partagée par les États-Unis qui ne souhaitent pas livrer leurs archives). Pourtant, l'image de mort (voir en ces pages la recension de l'ouvrage de Marc Crépon, Vivre avec) ramènerait à la conscience de la finitude, permettrait même de penser que quelquechose peut être sauvé de la chair des morts.

7 Ce que les Américains photographièrent à leur tour, lorsqu'ils occupèrent le territoire en septembre 1945, furent les destructions matérielles et les symptômes cliniques. Cela obéissait à une mission rationnelle et civilisatrice et permettait de connaître les effets réels, charnels des bombes. On essaie de tout voir, d'adopter une perspective générale et cavalière sur l'étendue de la dévastation, bref de garder mainmise et maîtrise de la conquête. On ne montre que le désert qui subsiste et les rares monuments qui pourraient servir de repères. Michael Lucken montre également qu'il existe, plus ou moins inconsciemment, une mémoire de la destruction et de sa prise de vue. En effet, le Japon avait déjà connu des tremblements de terre, des incendies ravageurs et on retrouve postérieurement les mêmes représentations à l'œuvre, des panoramas et des gestes muséaux identiques. Cela sert toujours à mieux domestiquer l'événement, la violence restant insoutenable.

8 Mais cette mémoire sert aussi de modèle pour la destruction, « on sait en effet que le souvenir des désastres subis par Tôkyô en 1923 et avant donnera à imaginer aux stratèges le pouvoir des bombes sur les villes de l'archipel» (p. 61). La dévastation y avait été vue et on pouvait de nouveau l'imaginer. Cela infirme la thèse de Gunther Anders (Hiroshima est partout, Paris, Éd. Le Seuil, 2008) sur le retard de l'imagination quant aux possibilités techniques : les Américains savaient très bien quel effet feraient leurs bombes. Les mémoires préfigurent les événements car ceux-ci s'inscrivent dans un réseau (d'inter-iconicité ?), mobilisé immédiatement après un choc. Un réflexe est à l'œuvre qui permet de conjurer la puissance d'un fait et de lui donner un sens, même falsifié. Si la photographie est susceptible de construire autrement les mémoires, c'est que le Japon en a aussi besoin aujourd'hui. Sur cet événement s'est cristallisé de fausses idées, s'est incarnée comme une sorte de masque où grimacent les victimes, les kamikazes, un sentiment de honte et de défaite. Ne semble venir du Japon que l'hyperviolence des jeux et des mangas, d'un désordre post- traumatique auquel on oppose notre sérénité rationnelle. On oublie les aspects sentimentaux, burlesques ou populaires de ce pays. 
9 Le processus de reconstitution de l'événement semble prendre forme dans la multiplication des médiations permettant de l'approcher. Michael Lucken analyse les meilleures photographies de Yamahata - Philippe Forest (Sarinagara, Paris, Gallimard, 2004) l'avait magistralement précédé dans cette tâche - où l'on voit, derrière le sentiment d'être descendus aux Enfers, la possibilité de l'espoir, de la fraternité, de la maternité, qui reprennent (un enfant porte son frère, une mère allaite son enfant), mais surtout, il met en correspondance photos et dessins de la même scène. Le photographe capte un peintre au travail (Yamada Eiji), chacun compose différemment, avec ses réflexes esthétiques : le premier donne à voir l'espace tout autour, y noie les cadavres que le second révèle dans leurs postures. On a le cadre et les détails de la mort, le tout et la partie, l'enregistrement scientifique et l'émotion: "On se rend compte que même à Nagasaki, même face à une expérience « à la limite ", celui qui veut voir ne peut échapper aux jeux de composition et aux effets de miroirs, comme si c'était la seule manière de rester debout. À peine a-t-il découvert le drame que le témoin actif est déjà dans la posture du narrateur » (p. 140).

10 Peut-être y a-t-il dans cette juxtaposition de la photographie et de l'illustration la modalité de compréhension du travail de la mémoire. Celle-ci doit sans cesse osciller entre le général et le particulier, peut-être même que chronologiquement elle agit ainsi : elle se veut englobante et cherche d'abord à transcender l'événement, à le dépasser afin de maîtriser un drame dans toute sa dimension, pour ensuite se concentrer sur les indices et sur les empreintes minimales. jamais ce travail ne semble assuré de la qualité de sa réception car l'équilibre requis, le dosage recherché par l'artiste doit rencontrer les capacités subjectives de l'observateur. Mais c'est bien, comme y invite cet ouvrage, dans cette zone entre les affects, les représentations, les mémoires engrangées et l'objet matériel où s'incarne le drame, que l'événement peut être compris.

11 Ce travail vient utilement compléter les récits et interrogations philosophiques qui émergent sur l'emploi de la bombe nucléaire et sur la nouvelle ère qui a suivi. La philosophie, à part l'œuvre de Gunther Anders et la contribution d'Alain Brossat ( $L a$ paix barbare, Paris, Éd. L'Harmattan, 2001), ne s'est pas encore emparée d'Hiroshima et de Nagasaki (i.e. Tanguy Wuillème, "À l'ombre inhumaine de la bombe ", Drôle d'époque, 16, 2005, pp.15-29, consultable sur www.revuedrole.com) et l'on peut présenter le siècle sans s'attarder sur cette étape (aucune mention n'est faite de l'événement chez Alain Badiou, François Furet, Alain Finkielkraut, Peter Sloterdijk...). Les montres ne sont pas encore à l'heure et sans doute se sont-elles bloquées au regard de la fulgurance de l'explosion, ce qui continue à la sacraliser ou à la maintenir dans les plis des spectres. Si Michael Lucken repère l'analogie de la montre arrêtée et de la photographie elle-même, c'est qu'il y va de la tâche de la pensée de dépasser et l'indice et la nature éternelle de l'événement pour savoir ce qui s'est passé,ou plutôt pensé au Japon en ce milieu du XX' siècle. 


\section{AUTEURS}

TANGUY WUILLÈME

CREM, Nancy-Université

Tanguy.Wuilleme@univ-nancy2.fr 